\title{
ПОИСК ФУНКЦИОНАЛЬНО ЗНАЧИМЫХ ГЕНЕТИЧЕСКИХ ВАРИАНТОВ, АССОЦИИРОВАННЫХ С САХАРНЫМ ДИАБЕТОМ 2-ГО ТИПА, С ИСПОЛЬЗОВАНИЕМ БИОИНФОРМАТИЧЕСКИХ МЕТОДОВ
}

\author{
Полуновский В.В., Колесникова И.С., Решетников В.В., Ермоленко Н.А., Гунбин К.В.
}

Сахарный диабет 2 типа (СД2) является одной из острых мировых медико-социальных проблем. На сегодняшний день известно, что СД2 обусловлен как средовыми, так и генетическими факторами, его наследуемость оценивают от 30 до 70\%.

ЦЕЛЬ: состояла в разработке алгоритма выявления и приоритезации генетических вариантов для создания скрининговой панели для оценки предрасположенности к развитию СД2 и сочетанных патологий.

МАТЕРИАЛЫ И МЕТОДЫ: В качестве основного источника данных использовали GeneAtlas - наиболее крупный ресурс результатов полногеномных анализов ассоциаций (GWAS). Для анализа мы выбрали следующие признаки: E11 (инсулиннезависимый сахарный диабет), E78 (нарушения обмена липопротеидов и другие липидемии), 110 (эссенциальная гипертензия), диабетическая ретинопатия (отдельный признак и как пересечение признаков H36+Е11), одновременное сочетание показателей индекса массы тела и доли жировой массы тела, а также Е66 (ожирение). Отбор однонуклеотидных генетических вариантов (single nucleotide polymorphism, SNP), ассоциированных с признаками, производился по импутированным данным (качество импутации $\geq 0,8$ ) с порогом статистической значимости ассоциации $\mathrm{p} \leq 1 \mathrm{E}-7$ (порог для значения beta не использовался). Были отобраны SNP с максимальной частотой (по данным баз данных ЕхAC-03, gnomAD 2.1.1, Kaviar-2015.09.23, 1000 genomes-2015.04.13, ESP 6500, платформа ANNOVAR) $\geq 0,01$, SNP с нулевой частотой в европейских популяциях исключали из анализа. Как правило, статистическая значимость SNV не связана с биологической значимостью гена для заболевания. Поэтому для приоритизации генов, мы использовали весь доступный пласт информации о генах (биологические сети, связь ген - признак по данным HPO human phenotype ontology, eQTL и т.п.), тесно сцепленных с SNV.

Для функциональной аннотации SNP была произведена непараметрическая нормировка, учитывающая взаимосогласованность значений для следующих их характеристик: частота, угловой коэффициент beta (характеристика силы эффекта), уровень значимости, показатель консервативности FitCons, pLI-скор гаплонедостаточности, также для кодирующих - рейтинг на основе данных DB NSFP4.0, для некодирующих — PAFA-скор, рейтинг RegulomeDB, данных GTEx (при их наличии). После вычисляли суммарный взвешенный рейтинг — Weighted Median Rank (WMR). Для каждого из отобранных генов выбирали 1 SNP в кодирующей и 1 в регуляторной области (если были), каждый с максимальным WMR. Отобранные гены были проанализированы на предмет связи с метаболическими процессами, сигнальными путями и заболеваниями с помощью ресурса EnrichR.

PЕзУЛЬтАТЫ: был разработан биоинформатический алгоритм поиска и приоритизации генов и полиморфизмов, ассоциированных с предрасположенностью к заболеваниям. Данный алгоритм был апробирован по отношению к СД2 и коморбидным ему заболеваниям. Были отобраны значимые SNP в более чем 100 генах/регуляторных областях, связанных с СД2, его осложнениями и коморбидными заболеваниями.

Выводы: разработанный биоинформатический алгоритм отбора и приоритизации генов и SNP позволил разработать скрининговую панель для выявления и расчета риска развития СД2 и связанных с ним осложнений и коморбидных заболеваний.

КЛЮЧЕВЫЕ СЛОВА: сахарный диабет 2-го muna; single nucleotide polymorphism; биоинформатика; ген; генетический скрининг; GWAS. 